les régimes : $2.800 \mathrm{k}$. cal. E.M. par kg. A 5 semaines d'âge, les animaux ont été quantitativement restreints selon deux programmes pré-établis : l'un moins sévère $(25.000 \mathrm{~K}$.cal. par oiseau de 0 à 24 semaines), l'autre plus sévère $(23.000 \mathrm{~K}$.cal.). Chacun de ces niveaux de restriction était appliqué aux trois régimes, conduisant à un total de 6 traitements.

Durant les premières semaines de la période de croissance, le poids vif était comparativement réduit par les faibles taux protéiques. Le niveau de restriction avait une influence de plus en plus prononcée avec l'âge. Néanmoins, l'effet du taux protéique était encore apparent à 20 semaines d'âge.

Les régimes à plus faible taux protéique donnés pendant la croissance retardaient la maturité sexuelle et réduisaient la production d'œufs, le poids des œufs et le nombre de ces derniers pouvant être incubés. Par contraste, le niveau de restriction avait très peu d'effet sur ces caractères.

Un aliment unique (à 15,7 p. 100 de protéines) était distribué pendant la période de ponte. La consommation pendant cette période dépendait du taux protéique de l'aliment reçu pendant les premières semaines de croissance ; plus élevé était le taux protéique durant la période de début de croissance, plus importante la consommation pendant la période de ponte. Ceci restait vrai, mais moins prononcé, après six mois de ponte, lorsque la consommation journalière d'aliment eût été limitée à 120 grammes par poule. Ce phénomène peut être expliqué en majeure partie par les différences de ponte.

Le poids vif à la fin de la période de ponte (67 semaines) était directement proportionnel au taux protéique de la ration de croissance; les animaux ne compensaient pas leur retard de croissance.

La restriction calorique pendant la période de croissance était très bénéfique aux performances de ponte des naines, peut-être parce qu'elle limitait leur tendance prononcée à l'engraissement. Au contraire, une déficience protéique pendant les premières semaines de croissance causait une réduction légère, mais nette, de la production d'œufs.

\title{
RATIONS CONCENTRÉES POUR LES PONDEUSES NAINES
}

J.H. QUISENBERRY ( $\left.{ }^{1}\right)$

Texas $A$ and $M$ University, Department of Poultry

Sciences, College Station, Texas, 77843, (U.S.A.)

L'introduction récente sur une large échelle d'un gène de nanisme lié au sexe à hérédité simple dans les programmes de test de divers sélectionneurs de souches commerciales a attiré l'attention sur la taille corporelle comme moyen potentiel d'accroitre le rendement alimentaire des volailles. Les résultats de précédentes expériences à l'Université A et $\mathbf{M}$ du Texas ont suggéré que les pondeuses naines ont besoin d'un aliment plus concentré en éléments nutritifs que leurs sœurs non naines. Dans une expérience incluant des oiseaux non nains divisés en trois classes de poids, légers, moyens et lourds, à 20 semaines d'âge, les animaux de petite taille répondaient plus que les lourds à une augmentation de la concentration de leur aliment. Un autre indice de l'importance qu'il y a à nourrir les oiseaux légers différemment de ceux dépassant le seuil minimum de poids de 1300 grammes a été obtenu antérieurement à partir d'expériences comparant les performances d'animaux éclos au printemps ou à l'automne dans un climat chaud, ainsi qu'à partir des premier et second test de performance "gène-environnement " du Texas.

Deux expériences comparant les performances de ponte des animaux nains et non-nains ont été réalisées. Dans la première, deux régimes (16 et $17 \%$ protéines), avec le même rapport calories/protéines et un, deux ou trois oiseaux par cage de $25,4 \times 45,7 \mathrm{~cm}$ ont été comparés.

(') Nous remercions John W. Bradley, Bill H. Doran et John C. Fowler de leur collaboration. 
Le taux protéique avait pe u d'effet sur le poids corporel et la ponte par poule et par jour chez les pondeuses naines, mais la taille des œufs, le pourcentage de gros œufs et le rendement alimentaire étaient augmentés par le taux protéique le plus élevé. Les poules non-naines avaient une production d'œufs inférieure lorsque leur nombre par cage augmentait, tandis que les naines pondaient plus d'œufs lorsqu'elles étaient deux par cage.

Dans la seconde expérience, des rapports protéines/calories (\%/cal. Kg. E.P. de $22 / 2145$, 17 /2035, $16 / 1980$ et 14/1925) étaient comparés. Une amélioration significative de la production d'œufs et du rendement alimentaire des naines recevant la ration à 22 p. 100 de protéines était observée. La viabilité des naines décroissait avec le taux protéique, alors qu'elle allait en augmentant pour les non-naines. Avec lous les taux protéiques réunis, le rapport de la performance des naines à celle des non-naines était : pour le poids corporel, 70 p. 100 ; la ponte, 80 p. 100 ; le poids des œufs, 90 p. 100 ; la consommation journalière d'aliment, 75 p. 100; le rendement alimentaire, 95 p. 100 , et le taux de survie, 98 p. 100.

Ces résultats indiquent que les oiseaux nains requièrent une ration plus concentrée que les lignées non-naines courantes. Des tests supplémentaires sont nécessaires pour déterminer l'équilibre optimum entre les composants de la ration pour ce type de naine, si ce dernier doit être employé à grande échelle commerciale pour la ponte.

\title{
DONNÉES COMPLÉMENTAIRES SUR LES BESOINS NUTRITIONNELS
}

\author{
DE LA REPRODUCTRICE NAINE \\ "Vedette I.N.R.A. JV I5 " \\ J. guillaume \\ Station de Recherches avicoles, I.N.R.A., \\ 37 - Nouzilly (France)
}

Dans une expérience précédente, nous avons montré que, durant la production, la reproductrice naine "Vedette INRA $J V 15$ " a un besoin énergétique modéré (environ 290 K. cal. E.M. /jour) et qu'une augmentation du taux protidique au-dessus de 16 p. 100 n'améliore pas ses performances.

Dans l'expérience présente, nous avons utilisé 576 poulettes reproductrices $J V 15$ provenant d'un troupeau commercial de multiplication, pesant $1,69 \mathrm{~kg}$ à 17 semaines. A cet âge, elles furent réparties en quatre classes de poids d'effectif égal, puis un nombre égal provenant de chaque classe de poids fut distribué au hasard entre 6 lots expérimentaux.

Trois rations isocaloriques $(2750$ K.cal. E.M. $/ \mathrm{kg})$ furent testées. Elles contenaient 15,5 - 14,0 ou 12.5 p. 100 de protéines brutes (respectivement rations A, B et C). Les taux de lysine et d'acides aminés soufrés étaient maintenus constants à 4,50 et 3,85 p. 100 des protéines brutes respectivement. L'ingestion d'aliment était limitée à $120 \mathrm{gr}$. par jour (lots $\mathrm{A}, \mathrm{B}$ et $\mathrm{C}$ ) ou restreinte plus sévèrement à partir ilu troisième mois ce ponte. Cette restriction était accentuée jusqu'au sixième mois de ponte (lots $\mathrm{AR}, \mathrm{BR}$ et $\mathrm{CR}$ ). Les oiseaux étaient placés en cage de ponte individuelles équipées de mangeoires individuelles et d’abreuvoirs goutte à goutte. Ils recevaient leur quantité allouée d'aliment trois fois par semaine.

Seul le lot A a montré des performances satisfaisantes. Le taux protéique comme la restriction de nourriture avaient des effets hautement significatifs sur l'intensité de production et le poids des œufs $(p<0,01)$. Il n'y avait pas d'interaction significative entre ces deux facteurs. Parmi les classes de poids différentes, il y avait des différences significatives de production d'œufs $(p<0,01)$ mais pas de poids d'œufs. Les poules légères produisaient davantage d'œufs et consommaient moins. Elles avaient, d'autre part, un taux plus élevé de mortalité.

La restriction en énergie et en protéines avait des effets différents : une restriction d'énergie 